Научная статья

УДк 366.1

DOI https://doi.org/10.24866/VVSU/2073-3984/2021-3/062-078

Е. Б. Кметь ${ }^{1}$

М. А. Потапенко ${ }^{2}$

Владивостокский государственный университет экономики и сервиса

Владивосток. Россия

\title{
Методический подход к совершенствованию usability сайта предприятия
}

Usability сайта как один из самых важных факторов пользовательского опыта оказывает сильное влияние на электронный бизнес. Сайты электронной коммерции теряют почти половину своих потенциальных продаж по причине того, что клиенты не могут пользоваться сайтом. Другими словами, при лучшем usability сайт может увеличить свои текущие продажи на 79\%. У сайта может быть эстетичный, минималистичный дизайн, но, если пользователь не может выполнить свою задачу максимально просто, он уйдет с него, а компания потеряет своего клиента. Для совершенствования сайта был разработан методический подход, который включает такие элементы, как актуальность темы исследования, предмет, цели и задачи; перечень и характеристика методов и инструментов исследования; система показателей для сбора данных; процесс разработки предложений по совершенствованию usability сайта и поэтапное описание инструментария исследования; комплексное исследование старого и нового дизайна сайта; общая оценка usability старого и нового дизайна в сравнении; комплекс предложений по совершенствованию использования сайта. Методический подход представляет интерес, применим для любого бизнеса, специализирующегося на электронных продажах, и протестирован на основе эмпирического комплексного исследования в форме эвристической оценки, анализа данных веб-аналитики, качественного и количественного usability-тестирования и двух опросов, результаты которых позволили разработать комплекс предложений по совершенствованию usability сайта. Комплекс предложений сформирован на основе данных качественного (выводы) и количественного (сравнение показателей) исследований, эвристической оценки, данных веб-аналитики.

\footnotetext{
1 Кметь Елена Борисовна - канд. экон. наук, доцент кафедры международного маркетинга и торговли; e-mail: kmeteb@yandex.ru

2 Потапенко Марк Александрович - бакалавр 4 курса направления подготовки «Торговое дело», профиль «Маркетинг в торговой деятельности»; e-mail: mark.potapenko99@gmail.com 62
} 
Ключевые слова и словосочетания: пользователь, качественное и количественное usability-тестирование, usability, показатели количественного тестирования, вебаналитика, эвристическая оценка, опросы SEQ и SUS.

E.B. Kmet

M.A. Potapenko

Vladivostok State University of Economics and Service

Vladivostok. Russia

\section{Methodological approach to improving the usability of the enterprise website}

Website usability is one of the most important user experience factors that have a strong impact on e-business. E-commerce sites lose almost half of their potential sales because users can't use the site. In other words, with better usability, a site can increase its current sales by $79 \%$. The site may have an aesthetic, minimalistic design, but if the user cannot perform their task as simply as possible, they will leave it, and the company will lose its client. To improve the usability of the site, a methodological approach was developed, which includes such elements as: the relevance of the research topic, subject, goals and objectives; a list and characteristics of research methods and tools; a system of indicators for data collection; the process of developing proposals for improving the usability of the site and a step-by-step description of the research tools; a comprehensive study of the old and new site design; a general assessment of the usability of the old and new design in comparison; a set of proposals for improving the usability of the site. The methodological approach is of interest, applicable to any business specializing in electronic sales and tested on the basis of an empirical comprehensive study in the form of heuristic evaluation, analysis of web analytics data, qualitative and quantitative usability testing and two surveys, the results of which allowed us to develop a set of proposals for improving the usability of the site. The set of proposals is formed on the basis of data from qualitative (conclusions) and quantitative (comparison of indicators) research, heuristic evaluation, and web analytics data.

Keywords: user, qualitative and quantitative usability testing, quantitative testing indicators, web analytics, heuristic evaluation, SEQ and SUS surveys.

\section{Введение}

Современный мир живет в эпоху Интернета, важность которого нельзя недооценивать не только в повседневной жизни, но и в экономике и бизнесе [1]. Приведем некоторые факты из публичной статистики компании WebCanape на 2020 год:

- 59\% населения пользуются Интернетом;

- $81 \%$ населения пользуется Интернетом в России;

- в среднем россияне пользуются Интернетом 7 часов 17 минут каждый день [2].

Интернет, в свою очередь, напрямую связан с веб-сайтами - основной точкой контакта, соединяющей клиента с компанией в Интернете. Можно 
утверждать, что мнение клиентов о компании зависит от их восприятия сайта. Соответственно сайт должен быть сделан так, чтобы у клиентов формировалось хорошее отношение к компании и он легко мог достичь своей цели (совершить покупку). Ответственностью компании, в свою очередь, является создание максимально простого и удобного для пользователя пути к достижению этой цели, а для этого нужен хороший дизайн сайта. Если сайт имеет плохой и неудобный дизайн, то, вероятно, пользователь перейдет на другой сайт и принесет прибыль другой компании. Помимо удобства пользования есть еще некоторые аспекты дизайна: полезность, простота поиска, надежность, желаемость, доступность, ценность, но наиболее важным и влиятельным из них является именно usability сайта [3].

Целью данной статьи являются разработка методического подхода и совершенствование usability сайтов. Данный методический подход универсален, представляет интерес и применим для любого бизнеса, специализирующегося на электронных продажах.

\section{Предмет исследования, источниковая база и авторская позиция}

Предметом исследования является влияние usability на улучшение использования сайта.

Питер Морвилл в 2004 году выявил семь главных факторов дизайна сайта: полезность, удобство использования, простота поиска, надежность, желаемость, доступность, ценность [4]. Удобство пользования сайтом - один из самых важных факторов, который нужно учитывать при составлении дизайна сайта. По данным всемирной консалтинговой компании NN/g, сайты электронной коммерции теряют почти половину своих потенциальных продаж по причине того, что клиенты не могут пользоваться сайтом. Другими словами, при лучшем удобстве пользования сайт может увеличить свои текущие продажи на 79\% [5]. Если сайт будет иметь хорошую надежность или просто будет полезным с точки зрения целей пользователей, но при этом им будет неудобно пользоваться, высока вероятность того, что пользователь просто перейдет на другой сайт. Поэтому можно смело сделать вывод, что удобство пользования - самый важный фактор дизайна, оказывающий значительное влияние на прибыль компании, если компания осуществляет свою деятельность через Интернет.

Существуют разные методы исследования usability сайта: качественное и количественное usability-тестирование, опросы, анкетирование, эвристическая оценка, анализ данных веб-аналитики, А/В-тестирование, сортировка карточек, айтрекинговое тестирование, дневниковое исследование, фокусгруппы, интервью. Каждый из этих методов отличается своими особенностями, достоинствами и недостатками [6-10].

Автор придерживается позиции, что для исчерпывающего и наиболее доступного исследования usability необходимо провести:

1) эвристическую оценку экспертами, что даст хороший ориентир для дальнейших изменений в дизайне [11]; 
Е.Б. Кметь, М.А. Потапенко. Методический подход к совершенствованию usability сайта...

2) анализ данных веб-аналитики, который позволит составить портрет пользователей, определить целевые действия, совершаемые ими, и выявить гипотезы относительно usability сайта $[12 ; 13]$;

3) качественное usability-тестирование, которое даст наиболее исчерпывающую информацию касательно удобства пользования сайтом и позволит подтвердить или опровергнуть разные гипотезы [14-16];

4) количественное usability-тестирование, которое позволит измерить удобство пользования сайтом через конкретные показатели: время на выполнение задач, успех, количество ошибок, субъективное удовлетворение [17; 18].

Подобный комплекс исследования даст исчерпывающую информацию о usability сайта.

\section{Методы исследования, методологические принципы и инструменты}

Методический подход к совершенствованию usability протестирован на основе эмпирического комплексного исследования в форме эвристической оценки трех экспертов в области веб-дизайна, анализа данных веб-аналитики с помощью Яндекс.Метрики, качественного и количественного usabilityтестирования и двух опросов SUS и SEQ, оценивающих субъективное удовлетворение пользователями [19].

Предлагаемый методический подход включает следующие элементы:

- актуальность темы исследования, предмет, цели и задачи;

- перечень и характеристика методов и инструментов исследования;

- система показателей для сбора данных;

- процесс разработки предложений по совершенствованию usability сайта и поэтапное описание инструментария исследования;

- комплексное исследование старого и нового дизайна сайта;

- общая оценка usability старого и нового дизайна в сравнении;

- комплекс предложений по совершенствованию usability сайта.

Процесс разработки предложений по совершенствованию usability сайта представлен на рис. 1.

\section{Комплексное исследование старого дизайна сайта}

Анализ данных веб-аналитики первой версии сайта. В первую очередь для дальнейших исследований необходимо было определить возраст основных групп посетителей, для этого проведен обзор структуры основных его пользователей. Выделены три основные группы участников качественного и количественного usability-тестирования: первая группа - люди возраста от 25 до 34 лет, вторая группа - от 35 до 44 лет, третья группа - от 45 до 54 лет. В сумме эти три группы занимают почти больше $75 \%$ от всех посетителей сайта.

Анализ карты ссылок позволил подтвердить гипотезу о том, что на данном сайте пользователи заинтересованы в выполнении четырех основных задач: познакомиться с информацией о компании, выяснить контакты компании, изучить товар и оформить заявку на него, рассчитать стоимость ворот в калькуляторе и оформить заявку. Это также было необходимо выявить для дальнейшего usability-тестирования. 
Территория новых возможностей. Вестник ВГУЭС. 2021. № 3

Этап 1. Формирование комплекса методов для исследования и оценки usability

- Шаг 1.1. Обзор всех методов исследования usability.

- Шаг 1.2. Обоснованный выбор необходимого комплекса методов.

- Шаг 1.3. Обоснованный выбор определенных показателей для дальнейшего анализа.

\section{Этап 2. Исследование usability старой версии сайта}

- Шаг 2.1. Сбор показателей веб-аналитики

- Шаг 2.2. Проведение эмпирического исследования в форме качественного и количественного usability тестирования, а также в форме эвристической оценки специалистов в области веб-дизайна.

- Шаг 2.3. Сбор результатов двух опросов в конце каждого количественного юзабилити тестирования.

Этап 3. Анализ данных исследования старой версии сайта

- Шаг 3.1. Веб-аналитика различных показателей.

- Шаг 3.2. Формирование основных проблем usability сайта после проведения качественного юзабилити тестирования и эвристической оценки.

- Шаг 3.3. Формирование общих числовых данных исходя из количественного юзабилити тестирования.

- Шаг 3.4. Текстовая аналитика результатов двух опросов в конце каждого количественного юзабилити тестирования.

Этап 4. Создание новой версии сайта

- Шаг 4.1. Формирование основных выводов

- Шаг 4.2. Создание новой версии сайта с учетом выявленных проблем и выводов

\section{Этап 5. Исследование и анализ usability новой версии сайта}

- Шаг 5.1. Проведение эмпирического исследования в форме качественного usability-тестирования и определение проблем и формирование выводов исследования.

- Шаг 5.2. Проведение эмпирического исследования в форме количественного usability тестирования и формирование общих числовых данных.

- Шаг 5.3. Текстовая аналитика результатов двух опросов в конце каждого количественного юзабилити тестирования.

\section{Этап 6. Оценка usability старой и новой версии сайта в сравнении}

- Шаг 6.1. Сравнение и оценка полученных данных по завершению качественного тестирования двух версий сайтов

- Шаг 6.2. Сравнение и оценка показателей двух версий сайта после проведения количественного юзабилити тестирования.

- Шаг 6.3. Общая оценка usability старой и новой версии сайта.

Рис. 1. Процесс разработки предложений по совершенствованию usability сайта

Анализ карты кликов позволил выявить некоторые гипотезы об удобстве пользования сайтом, например, частый клик на некликабельный прайс-лист, 66 
скорее всего, говорит о неудовлетворенности пользователей, которые, вероятно, хотели прочитать больше про товар или кликом оформить заявку на него.

Аналитика форм помогла выяснить, что форма подачи заявки на товар непонятна и неудобна пользователям, так как $87 \%$ посетителей никак не взаимодействовали с ней, а из 15 , которые пытались взаимодействовать, заполнил форму только один человек, процент конверсии составил $0,83 \%$.

В целом данные веб-аналитики помогли:

- определить портрет пользователей для дальнейших тестирований;

- подтвердить гипотезу о четырех основных выполняемых задачах пользователями на данном сайте;

- выявить некоторые предположения относительно удобства пользования сайтом.

Анализ данных эвристической оиенки экспертов первой версии сайта. Согласно рекомендациям консалтинговой компании NN/g, необходима была оценка трех экспертов для достоверного результата. Перед непосредственной оценкой дизайна были розданы десять основных эвристик, по которым и производилась оценка удобства пользования. Если подводить общий итог оценок всех трех экспертов, то наибольшие проблемы были обнаружены в следующих эвристиках [11;20]:

1) информированность о состоянии системы (непонятно название компании, чем она занимается);

2) схожесть системы с реальным миром (сайт очень перегружен разной информацией, некоторые ссылки и элементы сайта работают неочевидно и не приводят к желаемому результату);

3) свобода действий (перемещаться по сайту слишком сложно);

4) единообразие и стандарты (разные шрифты, разный дизайн некоторых страниц, разное неочевидное расположение некоторых элементов сайта, ошибки в тексте);

5) эстетичный и минималистичный дизайн (сайт перегружен информацией, плохие картинки, не связанные с тематикой сайта).

Анализ данных качественного иsability-тестирования первой версии сай$m a$. Необходимо было протестировать пять человек: два человека из возрастной группы от 25 до 34 лет, 2 человека - от 35 до 44 лет и 1 человек - от 45 до 54 лет. Тестирование проводилось на стандартном ультрабуке. В ходе тестирования участникам необходимо было соблюдать метод СТА, мышление вслух $[20 ; 21]$.

Участникам тестирования необходимо было выполнить следующие задачи:

1) выяснить название компании и что она продает;

2) выяснить контакты компании;

3) оформить заявку на три конкретных товара;

4) рассчитать стоимость и оформить заявку в калькуляторе ворот.

Суть данного исследования заключается в тщательном наблюдении за поведением пользователя, что ему удобно, а что нет, как он размышляет, когда соби- 
рается выполнять ту или иную задачу, куда смотрит в первую очередь, куда кликает.

Результаты качественного usability-тестирования представлены в табл. 1.

Таблииа 1

\section{Результаты качественного usability-тестирования старой версии сайта}

\begin{tabular}{|c|c|c|c|}
\hline Задание 1 & Задание 2 & Задание 3 & Задание 4 \\
\hline Проблемы & Проблемы & Проблемы & Проблемы \\
\hline $\begin{array}{l}\text { Много разных на- } \\
\text { званий компании }\end{array}$ & $\begin{array}{l}\text { В целом проблем не } \\
\text { было, так как задача } \\
\text { сама по себе про- } \\
\text { стая }\end{array}$ & $\begin{array}{l}\text { Мелкий и некликабель- } \\
\text { ный прайс-лист }\end{array}$ & $\begin{array}{l}\text { Непонятное располо- } \\
\text { жение калькулятора }\end{array}$ \\
\hline $\begin{array}{l}\text { Разные шрифты, } \\
\text { отсутствие единого } \\
\text { стиля оформления } \\
\text { внешнего вида }\end{array}$ & & $\begin{array}{l}\text { Отсутствие системы } \\
\text { автоматизированного } \\
\text { расчета стоимости това- } \\
\text { ров }\end{array}$ & $\begin{array}{l}\text { Расположение каль- } \\
\text { кулятора сверху вниз } \\
\text { затрудняло его ис- } \\
\text { пользование }\end{array}$ \\
\hline \multirow[t]{2}{*}{$\begin{array}{l}\text { Много разной ин- } \\
\text { формации }\end{array}$} & & $\begin{array}{l}\text { Неудобная и непонятная } \\
\text { пользователям система } \\
\text { оформления заказа }\end{array}$ & \multirow{2}{*}{$\begin{array}{l}\text { Непонятный пользо- } \\
\text { вателям дизайн (де- } \\
\text { монстрирующие кар- } \\
\text { тинки похожи на бан- } \\
\text { нер) }\end{array}$} \\
\hline & & Поиск не ищет товары & \\
\hline $\begin{array}{l}\text { Выводы: пользова- } \\
\text { телям было сложно } \\
\text { найти название ком- } \\
\text { пании и определить, } \\
\text { чем она занимается }\end{array}$ & $\begin{array}{l}\text { Выводы: в основ- } \\
\text { ном проблем с вы- } \\
\text { полнением и удоб- } \\
\text { ством не обнаруже- } \\
\text { но }\end{array}$ & $\begin{array}{l}\text { Выводы: пользователям } \\
\text { было очень сложно ре- } \\
\text { шить эту задачу, по } \\
\text { итогу никто с ней не } \\
\text { справился }\end{array}$ & $\begin{array}{l}\text { Выводы: все пользо- } \\
\text { ватели справились с } \\
\text { заданием, но это было } \\
\text { весьма затруднитель- } \\
\text { но и неудобно для них }\end{array}$ \\
\hline
\end{tabular}

Примечание: разработано авторами.

Результаты качественного тестирования выявили все проблемы, влияющие на usability.

Анализ данных количественного иsability-тестирования первой версии сайта.

Согласно рекомендациям консалтинговой компании $\mathrm{NN} / \mathrm{g}$, для получения достоверных результатов необходимо протестировать 20 участников [14]. Суть исследования заключается в сборе таких количественных показателей, как время на выполнение задач, процент успеха, количество ошибок. Субъективное удовлетворение - данный вид исследования позволяет измерить usability сайта для оценки последующих версий дизайна [22-25]. Пользователям нужно было выполнить те же самые задачи, что и в качественном тестировании. Данные количественного исследования первой версии сайта приведены в табл. 2. 
Е.Б. Кметь, М.А. Потапенко. Методический подход к совершенствованию usability сайта...

Таблиия 2

Результаты количественного тестирования старой версии сайта

\begin{tabular}{|c|c|c|c|c|c|}
\hline \multicolumn{6}{|c|}{ Измеряемый показатель - время (сек.) } \\
\hline & Задание 1 & Задание 2 & Задание 3 & Задание 4 & Общее время \\
\hline $\begin{array}{l}\text { Суммированные } \\
\text { значения }\end{array}$ & 1282 & 151 & 3600 & 2105 & 7138 \\
\hline Ср. арифм. & 64,1 & 7,55 & 180 & 105,25 & 89,2 \\
\hline \multicolumn{6}{|c|}{ Измеряемый показатель - процент успеха (\%) } \\
\hline & Задание 1 & Задание 2 & Задание 3 & Задание 4 & Общий \% успеха \\
\hline $\begin{array}{l}\text { Суммированные } \\
\text { значения }\end{array}$ & 30 & 100 & 0 & 100 & 58 \\
\hline \multicolumn{6}{|c|}{ Измеряемый показатель - ошибки (шт.) } \\
\hline & Задание 1 & Задание 2 & Задание 3 & Задание 4 & Всего \\
\hline $\begin{array}{l}\text { Суммированные } \\
\text { значения }\end{array}$ & 13 & 0 & 0 & 13 & 26 \\
\hline \multicolumn{6}{|c|}{ Измеряемый показатель - субъективное удовлетворение, SEQ (баллы) } \\
\hline & Задание 1 & Задание 2 & Задание 3 & Задание 4 & Сумма \\
\hline $\begin{array}{l}\text { Суммированные } \\
\text { значения }\end{array}$ & 97 & 127 & 57 & 74 & 97 \\
\hline Ср. знач. & 4,9 & 6,4 & 2,9 & 3,7 & \\
\hline $\begin{array}{l}\text { Процентное соот- } \\
\text { ношение, \% }\end{array}$ & 38 & 90 & 16 & 18 & 27 \\
\hline
\end{tabular}

Примечание: разработано авторами.

Измеряемый показатель - время.

В целом все задачи были выполнены очень медленно, относительно нормальный показатель только у второй задачи, тем не менее, при хорошем дизайне пользователь может выяснить контакты компании еще быстрее, потратив примерно 3 секунды. Среднее время выполнения первой задачи - 1 минута, это очень плохой результат для подобной задачи.

Измеряемый показатель - прочент успеха.

При выполнении первого задания показатель успеха составил $30 \%$, это означает, что 70\% пользователей не выполняют правильно поставленную задачу. 
Чаще всего пользователи называли неверное название компании, так как эта информация неочевидно представлена на сайте.

Никто не справился с третьим заданием. Был установлен лимит в 3 минуты. Если пользователь не справлялся с заданием менее чем за 3 минуты, то задача считалась невыполненной. Это говорит о том, что дизайн сайта очень плохо адаптирован к выполнению третьей задачи.

Все остальные задачи были выполнены, хотя четвертое задание выполнить пользователям было очень неудобно.

Измеряемый показатель - количество ошибок.

Допустимое количество ошибок в первом дизайне или нет, можно выяснить только при сравнении с количеством ошибок пользователей новой версии дизайна. За ошибку считалось:

- неправильный клик (клик, который не приводит к целевому действию);

- лишний скроллинг (излишняя работа колесиком мыши в поисках информации).

Измеряемый показатель - субъективное удовлетворение, SEQ.

Опрос SEQ состоит из одного вопроса: пользователям необходимо было оценить простоту выполнения каждого задания по 7-балльной шкале. В целом видно, что, по мнению пользователей, простота выполнения заданий крайне низкая, за исключением выполнения второго задания (причины этого изложены выше),

Измеряемый показатель - субъективное удовлетворение, SUS.

SUS-опрос включает десять вопросов, где пользователи по 5-балльной шкале оценивают удобство пользования сайтом. Средний балл SUS составил 39 баллов, что соответствует международной оценке «F». Пользователи оценили уровень usability сайта как критичный.

Итак, по всем показателям результаты usability сайта оказались очень плохими: слишком много времени у пользователей уходит на выполнение задач, маленький процент успеха выполнения задач и субъективного удовлетворения (пользователи недовольны сайтом), большое количество допускаемых ошибок (хотя это будет ясно только в сравнении с результатом другого дизайна).

\section{Комплекс предложений по совершенствованию usability сайта}

Проанализировав данные проблемы, можно принять решение о разработке нового дизайна сайта с целью улучшить его usability и устранить все выявленные ранее проблемы.

Приняты следующие решения:

- сделать дизайн максимально минималистичным и простым;

- в самом верху сайта выделить название компании с его логотипом;

- на главной странице простой фразой обозначить, чем занимается компания, чтобы пользователи сразу понимали, куда они попали;

- сделать простую и минималистичную шапку для наиболее легкого ориентирования по сайту; 
Е.Б. Кметь, М.А. Потапенко. Методический подход к совершенствованию usability сайта...

- разработать калькулятор товара и расположить его на главной странице, чтобы автоматизировать расчет стоимости товара и упростить задачу;

- поместить калькулятор ворот в шапку для быстрого его нахождения;

- видоизменить калькулятор ворот из вертикального вида в горизонтальный;

- в правом верхнем углу сайта поместить номер компании.

Далее будут представлены данные количественного и качественного usability-тестирования второй версии сайта.

Общая оценка usability старого и нового дизайна в сравнении.

Результаты качественного usability-тестирования новой версии сайта представлены в табл. 3.

Таблииа 3

Результаты качественного usability-тестирования новой версии сайта

\begin{tabular}{|c|c|c|c|}
\hline Задание 1 & Задание 2 & Задание 3 & Задание 4 \\
\hline Проблемы & Проблемы & Проблемы & Проблемы \\
\hline \multirow[t]{3}{*}{$\begin{array}{l}\text { Проблем не было } \\
\text { обнаружено }\end{array}$} & \multirow[t]{3}{*}{$\begin{array}{l}\text { Проблем не было } \\
\text { обнаружено }\end{array}$} & $\begin{array}{l}\text { Не все пользователи сра- } \\
\text { зу различали калькулятор } \\
\text { ворот от калькулятора } \\
\text { товара, соответственно } \\
\text { не сразу находили нуж- } \\
\text { ный калькулятор }\end{array}$ & $\begin{array}{l}\text { Калькулятор на некото- } \\
\text { рых мониторах был } \\
\text { слишком мелкий }\end{array}$ \\
\hline & & $\begin{array}{l}\text { Функция «добавить то- } \\
\text { вар» оказалась не очень } \\
\text { удобна и понятна неко- } \\
\text { торым пользователям }\end{array}$ & $\begin{array}{l}\text { Демонстрирующая кар- } \\
\text { тинка забора была очень } \\
\text { похожа на рекламный } \\
\text { баннер и дезориентиро- } \\
\text { вала пользователей }\end{array}$ \\
\hline & & $\begin{array}{l}\text { Большинство пользова- } \\
\text { телей заполняют данные } \\
\text { сверху вниз, а в кальку- } \\
\text { ляторе товара нужно } \\
\text { заполнять справа налево, } \\
\text { это не всем сразу стано- } \\
\text { вилось понятно }\end{array}$ & $\begin{array}{l}\text { Главное меню не всем } \\
\text { было заметно из-за цвета, } \\
\text { сливающегося с общим } \\
\text { фоном }\end{array}$ \\
\hline $\begin{array}{l}\text { Выводы: пользо- } \\
\text { вателям было } \\
\text { легко понять, чем } \\
\text { занимается ком- } \\
\text { пания, и прочесть } \\
\text { ее название, пото- } \\
\text { му что информа- } \\
\text { ция об этом была } \\
\text { явно выделена и } \\
\text { находилась на } \\
\text { главной странице }\end{array}$ & $\begin{array}{l}\text { Выводы: задача } \\
\text { также упростилась } \\
\text { за счет того, что } \\
\text { номер компании } \\
\text { был помещен в } \\
\text { верхний правый } \\
\text { угол }\end{array}$ & $\begin{array}{l}\text { Выводы: пользователям } \\
\text { было намного удобнее } \\
\text { решить эту задачу по } \\
\text { сравнению с предыдущим } \\
\text { дизайном, но есть некото- } \\
\text { рые недочеты }\end{array}$ & $\begin{array}{l}\text { Выводы: все пользователи } \\
\text { справились с заданием, и } \\
\text { это было для них гораздо } \\
\text { легче в сравнении с пре- } \\
\text { дыдущим дизайном }\end{array}$ \\
\hline
\end{tabular}

Примечание: разработано авторами. 
Территория новых возможностей. Вестник ВГУЭС. 2021. № 3

Результаты количественного usability-тестирования новой версии сайта представлены в табл. 4.

Таблища 4

Результаты количественного тестирования новой версии сайта

\begin{tabular}{|c|c|c|c|c|c|}
\hline \multicolumn{6}{|c|}{ Измеряемый показатель - время (сек.) } \\
\hline & Задание 1 & Задание 2 & Задание 3 & Задание 4 & $\begin{array}{c}\text { Общее } \\
\text { время }\end{array}$ \\
\hline $\begin{array}{l}\text { Суммирован- } \\
\text { ные значения }\end{array}$ & 20 & 60 & 2488 & 1208 & 3776 \\
\hline Ср. ариф. & 1 & 3 & 124,4 & 60,4 & 47,2 \\
\hline \multicolumn{6}{|c|}{ Измеряемый показатель - процент успеха (\%) } \\
\hline & Задание 1 & Задание 2 & Задание 3 & Задание 4 & $\begin{array}{c}\text { Общий \% } \\
\text { успеха }\end{array}$ \\
\hline $\begin{array}{l}\text { Суммирован- } \\
\text { ные значения }\end{array}$ & 100 & 100 & 80 & 100 & 95 \\
\hline \multicolumn{6}{|c|}{ Измеряемый показатель - ошибки (шт.) } \\
\hline & Задание 1 & Задание 2 & Задание 3 & Задание 4 & Всего \\
\hline $\begin{array}{l}\text { Суммирован- } \\
\text { ные значения }\end{array}$ & 0 & 0 & 32 & 4 & 36 \\
\hline \multicolumn{6}{|c|}{ Измеряемый показатель - субъективное удовлетворение, SEQ (баллы) } \\
\hline & Задание 1 & Задание 2 & Задание 3 & Задание 4 & Сумма \\
\hline $\begin{array}{l}\text { Суммирован- } \\
\text { ные значения }\end{array}$ & 138 & 136 & 121 & 133 & 355 \\
\hline Ср. знач. & 6,9 & 6,8 & 6,1 & 6,7 & \\
\hline $\begin{array}{l}\text { Процентное } \\
\text { соотношение, } \\
\%\end{array}$ & 100 & 98 & 80 & 96 & 94 \\
\hline
\end{tabular}

Примечание: разработано авторами.

Измеряемый показатель - время.

Среднее время на выполнение первой задачи - 1 сек., по сравнению со временем выполнения при первом дизайне результат улучшился на $6310 \%$.

Среднее время на выполнение второй задачи - 3 сек., шапка сайта состоит всего из трех пунктов, среди которых пункт «контакты», при этом длинный номер компании находится в правом верхнем углу и всегда на виду у пользовате- 
лей. По сравнению с временем выполнения при первом дизайне результат улучшился на $152 \%$.

Среднее время на выполнение третьей задачи - 2 мин., это на $45 \%$ быстрее, при условии, что фактически на выполнение этой задачи при первом дизайне пользователи тратили больше 3 минут (эти результаты не записывались, так как был установленный максимум по времени - 3 минуты).

Среднее время на выполнение четвертой задачи - 1 мин., это на 74\% быстрее, чем время, которое тратили пользователи при первом дизайне. Такого результата удалось достичь благодаря изменениям, о которых говорилось выше.

Процент улучшения выполнения всех четырех задач относительно времени в сравнении с первым дизайном сайта составил $175 \%$. На $175 \%$ пользователи быстрее начали справляться с задачами на второй версии сайта.

Измеряемый показатель - процент успеха.

При выполнении первой задачи процент успеха составил 100\%, что на $70 \%$ больше, чем при первом дизайне.

При выполнении второй задачи процент успеха составил 100\%, как в первой версии сайта.

При выполнении третьей задачи процент успеха составил $-80 \%$, это на $80 \%$ больше, чем при первом дизайне сайта, когда с этой задачей никто не справился.

При выполнении четвертой задачи процент успеха составил 100\%, как и в первой версии сайта.

Процент улучшения успеха при выполнении всех четырех задач - 37\% и в целом составил $95 \%$.

Измеряемый показатель - ошибки.

При выполнении первой задачи процент количества ошибок - 0, что на 13 меньше, чем в предыдущем дизайне, т.е. на $1300 \%$ больше.

При выполнении второй задачи процент успеха составил - 0, точно такой же результат, как и в первом дизайне, так как задача весьма простая сама по себе.

При выполнении третьей задачи процент успеха составил - 32. В этом случае отсутствует необходимость сравнивать количество ошибок с первой версией, поскольку третья задача не была выполнена в принципе. Это не очень хороший результат, свидетельствующий о необходимости менять калькулятор под пользователя.

При выполнении четвертой задачи процент успеха составил 4, это на 9 ошибок меньше, чем было всего допущено в первом дизайне.

Процент уменьшения ошибок при выполнении всех четырех задач $-550 \%$.

Измеряемьій показатель - субъективное удовлетворение, SEQ.

Процент простоты выполнения первой задачи, по мнению пользователей, составил $100 \%$, т.е. клиенты получили максимальное удовлетворение от пользо- 
вания сайтом. На $62 \%$ этот показатель увеличился по сравнению с первой версией сайта.

Процент простоты выполнения второй задачи, по мнению пользователей, составил 98\%, т.е. этот показатель увеличился по сравнению с первой версией сайта на $8 \%$. Такой небольшой процент обусловлен простотой задачи.

Процент простоты выполнения третьей задачи, по мнению пользователей, составил 80\%. Этот показатель увеличился по сравнению с первой версией сайта на $64 \%$.

Процент простоты выполнения четвертой задачи, по мнению пользователей, составил 96\%. Данный показатель увеличился по сравнению с первой версией сайта на $78 \%$.

Процент улучшения субъективного удовлетворения после пользования сайта $-67 \%$, в целом составляет $94 \%$. Это говорит о том, что, по мнению пользователей, все четыре задачи на сайте выполняются для них легко.

Измеряемый показатель - субъективное удовлетворение, SUS.

Оценка удобства от пользования данной версией сайта, по мнению пользователей, составила 89 , что на $128 \%$ больше, чем в первой версии. Данная оценка соответствует самой максимальной оценке удобства пользования.

Результаты сравнительной характеристики данных количественного тестирования старой и новой версии сайта представлены в табл. 5.

Таблица 5

\section{Результаты сравнительной характеристики данных количественного тестирования старой и новой версии сайта}

\begin{tabular}{|l|c|c|c|c|c|}
\hline \multicolumn{7}{|c|}{ Измеряемый показатель - время (сек.) } \\
\hline \multirow{2}{*}{ Версия } & Задача 1 & Задача 2 & Задача 3 & Задача 4 & Общее время \\
\hline 1 & 1282 & 151 & 3600 & 2105 & 3538 \\
\hline 2 & 20 & 60 & 2488 & 1208 & 1288 \\
\hline Улучшение, \% & $6310 \%$ & $152 \%$ & $45 \%$ & $74 \%$ & $175 \%$ \\
\hline \multirow{2}{*}{ Версия } & Измеряемый показатель - процент успеха (\%) & \\
\hline 1 & Задача 1 & Задача 2 & Задача 3 & Задача 4 & Общий успех \\
\hline 2 & 30 & 100 & 0 & 100 & 58 \\
\hline Улучшение & 700 & 100 & 80 & 100 & 95 \\
\hline
\end{tabular}


Е.Б. Кметь, М.А. Потапенко. Методический подход к совершенствованию usability сайта...

Окончание табл. 5

\begin{tabular}{|l|c|c|c|c|c|}
\hline \multicolumn{7}{|c|}{ Измеряемый показатель - ошибки (шт.) } \\
\hline \multirow{2}{*}{ Версия } & Задача 1 & Задача 2 & Задача 3 & Задача 4 & Общие ошибки \\
\hline 1 & 13 & 0 & 0 & 13 & 26 \\
\hline 2 & 0 & 0 & 0 & 4 & 4 \\
\hline Улучшение & $1300 \%$ & $0 \%$ & $0 \%$ & $225 \%$ & $550 \%$ \\
\hline \multicolumn{2}{|c|}{ Измеряемый показатель - субъективное удовлетворение, SEQ (баллы) } \\
\hline \multicolumn{1}{|c|}{ Версия } & Задача 1 & Задача 2 & Задача 3 & Задача 4 & Общий процент \\
\hline 1 & $38 \%$ & $90 \%$ & $16 \%$ & $18 \%$ & $27 \%$ \\
\hline 2 & $100 \%$ & $98 \%$ & $80 \%$ & $96 \%$ & $94 \%$ \\
\hline Улучшение & $62 \%$ & $8 \%$ & $64 \%$ & $78 \%$ & $67 \%$ \\
\hline
\end{tabular}

Примечание: разработано авторами.

Общее улучшение usability представлено в табл. 6.

Таблица 6

Общая оценка usability сайта в сравнении

\begin{tabular}{|c|c|c|c|c|c|}
\hline \multicolumn{7}{|c|}{ Общая оценка usability } \\
\hline Версия & Время & Успех & Ошибки & $\begin{array}{c}\text { Субъективное } \\
\text { удовлетворение }\end{array}$ & $\begin{array}{c}\text { Общее улучшение } \\
\text { usability }\end{array}$ \\
\hline 1 & 0 & 0 & 0 & 0 & 0 \\
\hline 2 & $+175 \%$ & $+37 \%$ & $+550 \%$ & $+128 \%$ & $+173 \%$ \\
\hline
\end{tabular}

Примечание: разработано авторами.

Общая оценка улучшения usability была рассчитана как среднегеометрическое всех четырех показателей: время, успех, ошибки, субъективное удовлетворение - и составила $173 \%$, то есть удобство пользованием сайтом в новом его дизайне выросло в общем на 173\% по сравнению с предыдущим [26].

\section{Выводы и научная новизна}

Метод совершенствования usability был разработан теоретически и протестирован эмпирически через комплекс методов исследования usability. В совокупности методы дали достоверные и исчерпывающие данные об удобстве пользования первой версии сайта, что позволило сформулировать определенный комплекс предложений по совершенствованию usability сайта. Далее была про- 
тестирована вторая версия сайта, и благодаря исходным данным можно сделать вывод, что комплекс предложений был правильный, ведь вторая версия сайта оказалась более удобной в использовании. В ходе данного исследования также выявлены некоторые ошибки в новом дизайне сайта, которые необходимо исправить в последующей работе.

Научная новизна исследования заключается в разработке конкретного комплекса методов исследования и совершенствования usability сайта, который был проверен эмпирическим путем. Данная методика универсальна и проста в реализации, подходит к исследованию и совершенствованию любого сайта.

1. Кметь Е. Б. Маркетинговые коммуникации. Теория, практика, управление: учебник для магистров. - Саратов: Ай Пи Эр Медиа, 2016. - 183 с.

2. Вся статистика Интернета на 2020 год - цифры и тренды в мире и в России. - Текст: электронный // WebCanape: [сайт]. - URL: https://www.web-canape.ru/business/internet2020-globalnaya-statistika-i-trendy/ (дата обращения: 24.12.2020).

3. Nielsen J. User interface directions for the web. - Communications of the ACM, 1999. $218 \mathrm{c}$.

4. Morville P. User Experience Design. - Текст: электронный // Semantic studios: [сайт]. URL: http://semanticstudios.com/user_experience_design/ (дата обращения: 24.12.2020).

5. Nielsen J. Is Poor Usability Killing E-Commerce. - Текст: электронный // NNgroup: [сайт]. - URL: https://www.nngroup.com/articles/did-poor-usability-kill-e-commerce/ (дата обращения: 15.12 .2020$)$.

6. Кметь Е.Б., Чжипэн Ч., Балгум Л. Оценка юзабилити сайтов университетских библиотек КНР. - Текст: электронный // Трансграничные рынки товаров и услуг: проблемы исследования: материалы III Междунар. науч.-практ. конф., г. Владивосток, 7-8 ноября 2019 г. / Дальневост. федер. ун-т; Школа экономики и менеджмента; отв. ред. И. М. Романова. - Электрон. дан. - Владивосток: Изд-во Дальневост. федер. ун-та, 2019. - C. 108-112.

7. Nielsen J. Putting A/B Testing in Its Place. - Текст: электронный // NNgroup: [сайт]. URL: https://www.nngroup.com/articles/putting-ab-testing-in-its-place/ (дата обращения: 07.01.2021)

8. Pernice K. User Interviews: How, When, and Why to Conduct Them. - Текст: электронный // NNgroup: [сайт]. - URL: https://www.nngroup.com/articles/interviewing-users/ (дата обращения: 07.01.2021).

9. Nielsen J. The Use and Misuse of Focus Groups. - Текст: электронный // NNgroup: [сайт]. - 1997. - URL: https://www.nngroup.com/articles/focus-groups/ (дата обращения: 07.01.2021).

10. Budiu R. Quantitative vs. Qualitative Usability Testing. - Текст: электронный // NNgroup: [сайт]. - 2017. - URL: https://www.nngroup.com/articles/quant-vs-qual/ (дата обращения: 07.01.2021).

11. Nielsen J. Iterative User Interface Design. - Текст: электронный // NNgroup: [сайт]. 1993. - URL: https://www.nngroup.com/articles/iterative-design/ (дата обращения: 10.01.2021).

12. Cardello J. Three Uses for Analytics in User-Experience Practice. - Текст: электронный // NNgroup: [сайт]. - 2013. - URL: https://www.nngroup.com/articles/analytics-userexperience/ (дата обращения: 10.01.2021). 
Е.Б. Кметь, М.А. Потапенко. Методический подход к совершенствованию usability сайта...

13. Torres A. Analytics for UX Designers: Best Practices \& Tools. - Текст: электронный // QuantumMetric: [сайт]. - 2013. - URL: https://www.quantummetric.com/blog/analyticsfor-ux-designers-and-data-driven-design/ (дата обращения: 07.01.2021).

14. Moran K. Usability Testing 101. - Текст: электронный // NNgroup: [сайт]. - 2019. URL: https://www.nngroup.com/articles/usability-testing-101/

15. Moran K. Writing Tasks for Quantitative and Qualitative Usability Studies. - Текст: электронный // NNgroup: [сайт]. - 2018. - URL: https://www.nngroup.com/articles/testtasks-quant-qualitative/ (дата обращения: 10.01.2021).

16. Nielsen J. Time Budgets for Usability Sessions. - Текст: электронный // NNgroup: [сайт]. - 2005. - URL: https://www.nngroup.com/articles/time-budgets-for-usabilitysessions/ (дата обращения: 07.01.2021).

17. Loranger H. Checklist for Planning Usability Studies. - Текст: электронный // NNgroup: [сайт]. - 2016. - URL: https://www.nngroup.com/articles/usability-test-checklist/ (дата обращения: 10.01.2021).

18. Schade A. Avoid Leading Questions to Get Better Insights from Participants. - Текст: электронный // NNgroup: [сайт].- 2017. - URL: https://www.nngroup.com/articles/leading-questions/ (дата обращения: 10.01.2021).

19. Laubheimer P. Beyond the NPS: Measuring Perceived Usability with the SUS, NASATLX, and the Single Ease Question After Tasks and Usability Tests. - Текст: электронный // NNgroup: [сайт]. - 2018. - URL: https://www.nngroup.com/articles/measuringperceived-usability/ (дата обращения: 10.01.2021).

20. Nielsen J. 10 Usability Heuristics for User Interface Design. - Текст: электронный // NNgroup: [сайт]. - 2020. - URL: https://www.nngroup.com/articles/ten-usabilityheuristics/ (дата обращения: 15.12.2020).

21. Bergstrom J.R. Usability Testing for Survey Research. - Morgan Kaufmann, 2017. - 218 c.

22. Nielsen J. Success Rate: The Simplest Usability Metric. - Текст: электронный // NNgroup: [сайт]. - 2001. - URL: https://www.nngroup.com/articles/success-rate-thesimplest-usability-metric/ (дата обращения: 15.12.2020).

23. Kumar S. 3 Key Metrics to Identify the Usability of Your Product. - Текст: электронный // DZone: [сайт]. - 2019. - URL: https://dzone.com/articles/three-keymetrics-of-product-usability (дата обращения: 10.01.2021).

24. Nielsen J. Usability Metrics. - Текст: электронный // NNgroup: [сайт]. - 2001. - URL: https://www.nngroup.com/articles/usability-metrics/ (дата обращения: 10.01.2021).

25. Sauro J. Measuring errors in the user experience. - Текст: электронный // MeasuringU: [сайт]. - 2012. - URL: https://clck.ru/T2fPf (дата обращения: 10.01.2021).

26. Nielsen J. Iterative User Interface Design // IEEE Computer Society. - Washington, 1993. Iss. 26. - P. 32-41.

\section{References}

1. Kmet' E. B. Marketingovye kommunikacii. Teoriya, praktika, upravlenie: uchebnik dlya magistrov. - Saratov: Aj Pi Er Media, 2016. - 183 s. (uchebnye materialy NE razmeshchayut $\mathrm{v}$ spiskah nauchnyh statej)

2. Vsya statistika Interneta na 2020 god - cifry i trendy v mire i v Rossii. - Tekst: elektronnyj // WebCanape: [sajt]. - URL: https://www.web-canape.ru/business/internet-2020globalnaya-statistika-i-trendy/ (data obrashcheniya: 24.12.2020).

3. Kmet' E. B., Chzhipen CH., Balgum L. Ocenka yuzabiliti sajtov universitetskih bibliotek KNR. - Tekst: elektronnyj // Transgranichnye rynki tovarov i uslug: problemy issledovaniya: materialy III Mezhdunar. nauch.-prakt. konf., g. Vladivostok, 7-8 noyabrya 2019 g. / Dal'nevost. feder. un-t; SHkola ekonomiki i menedzhmenta; otv. red. 
I. M. Romanova. - Elektron. dan. - Vladivostok: Izd-vo Dal'nevost. feder. un-ta, 2019. S. $108-112$.

(C) Е.Б. Кметь, 2021

(C) М.А. Потапенко, 2021

Для цитирования: Кметь Е.Б., Потапенко М.А. Методический подход к совершенствованию usability сайта предприятия // Территория новых возможностей. Вестник Владивостокского государственного университета экономики и сервиса. - 2021. - Т. 13, № 3. - C. 62-78.

For citation: Kmet E.B., Potapenko M.A. Methodological approach to improving the usability of the enterprise website, The Territory of New Opportunities. The Herald of Vladivostok State University of Economics and Service, 2021, Vol. 13, № 3, pp. 62-78.

DOI https://doi.org/10.24866/VVSU/2073-3984/2021-3/062-078

Дата поступления: 22.06.2021. 